\title{
ON THE SURFACES OF COINCIDENCE
}

\section{SU-CHENG CHANG}

The purpose of this note is to give a characteristic property of the surfaces of coincidence. We show first that there are six and only six asymptotic osculating quadrics $Q_{j}(j=1, \cdots, 6)$ associated with a point $P$ of a non-ruled analytic surface $(M)$ each of which contains the four consecutive asymptotic tangents $t_{i(j)}(i=1,2,3,4)$ of one system along a curve $T$ through $P$ and on the surface $(M)$. As the curve $T$ must be tangent to a direction of Darboux at $P$, it is further proved that each quadric $Q_{i}$ is an osculating quadric of an asymptotic ruled surface $R$ along a curve of Darboux when and only when the surface $(M)$ is a surface of coincidence.

Let the surface $(M)$ be referred to its asymptotic parameters $u, \tau$, and let $\left\{M, M_{1}, M_{2}, M_{3}\right\}$ be the normal tetrahedron of Cartan, $M_{1} M$ and $M_{2} M$ being the two asymptotic tangents and $M M_{3}, M_{1} M_{2}$ the directrices of Wilczynski. Then the surface $(M)$ is, except for a projective transformation, determined by the following system of equations:

(1)

$$
\left\{\begin{array}{l}
\frac{\partial M}{\partial u}=\frac{1}{2} \frac{\partial \log \gamma}{\partial u} M+M_{1}, \frac{\partial M_{1}}{\partial u}=B^{2} M-\frac{1}{2} \frac{\partial \log \gamma}{\partial u} M_{1}+\beta M_{2} \\
\frac{\partial M_{2}}{\partial u}=\frac{h_{1}}{2} M+\frac{1}{2} \frac{\partial \log \gamma}{\partial u} M_{2}+M_{3} \\
\frac{\partial M_{3}}{\partial u}=A^{2} \beta M+\frac{1}{2} h_{1} M_{1}+B^{2} M_{2}+\frac{-1}{2} \frac{\partial \log \gamma}{\partial u} M_{3} ; \\
\frac{\partial M}{\partial v}=\frac{1}{2} \frac{\partial \log \beta}{\partial v} M+M_{2}, \frac{\partial M_{2}}{\partial v}=A^{2} M-\frac{1}{2} \frac{\partial \log \beta}{\partial v} M_{2}+\gamma M_{1} \\
\frac{\partial M_{1}}{\partial v}=\frac{k_{1}}{2} M+\frac{1}{2} \frac{\partial \log \beta}{\partial v} M_{2}+M_{3}, \\
\frac{\partial M_{3}}{\partial v}=B^{2} \gamma M+A^{2} M_{1}+\frac{1}{2} k_{1} M_{2}-\frac{1}{2} \frac{\partial \log \beta}{\partial v} M_{3} ;
\end{array}\right.
$$

where $M$ denotes a point of the surface with coordinates $M^{i}$ $(i=1,2,3,4)$ and

$$
h_{1}=\beta \gamma-(\log \beta)^{\prime \prime}, \quad k_{1}=\beta \gamma-(\log \gamma)^{\prime \prime} .
$$

The integrability conditions of the system (1) are

Received by the editors April 24, 1943. 
(2)

$$
\begin{gathered}
\frac{\partial A^{2}}{\partial u}=\frac{h_{1}}{2} \frac{\partial\left(\log \beta h_{1}\right)}{\partial v}, \quad \frac{\partial B^{2}}{\partial v}=\frac{k_{1}}{2} \frac{\partial\left(\log \gamma k_{1}\right)}{\partial u}, \\
A \frac{\partial(A \beta)}{\partial v}=B \frac{\partial(B \gamma)}{\partial u} .
\end{gathered}
$$

Following Godeaux, ${ }^{1}$ we denote by $\cdots, U_{n}, \cdots, U_{1}, U, V, V_{1}$, $\ldots, V_{n}, \ldots$ the self-polar Laplace sequence in $S_{5}$, where $U=\left|M, M_{1}\right|$ and $V=\left|M, M_{2}\right|$ are the images in $S_{5}$ of the two asymptotic tangents of the surface $(M)$ at $M$ respectively. It is well known that ${ }^{2}$

$$
\begin{gathered}
U^{10}=\beta V, \quad V^{01}=\gamma U ; \\
\left\{\begin{array}{c}
U_{n}^{01}=U_{n+1}+U_{n}\left(\log \beta h_{1} \cdots h_{n}\right)^{01}, \\
U_{n}^{10}=h_{n} U_{n-1}, \\
h_{n}=h_{n-1}-\left(\log \beta h_{1} \cdots h_{n}\right)^{11}
\end{array}\right.
\end{gathered}
$$

and

$$
\left\{\begin{aligned}
V_{n}^{10} & =V_{n+1}+V_{n}\left(\log \gamma k_{1} \cdots k_{n}\right)^{10}, \\
V_{n}^{01} & =k_{n} V_{n-1}, \\
k_{n} & =k_{n-1}-\left(\log \gamma k_{1} \cdots k_{n}\right)^{11},
\end{aligned}\right.
$$

where ()$^{i j}$ denotes the partial derivative of order $i+j$ formed by differentiating ( ) $i$ times with respect to $u$ and $j$ times with respect to $v$.

Since seven points in $S_{5}$ are always dependent, we find that

$$
\begin{aligned}
U_{3} & +\left(\log \beta^{3} h_{1}^{2} h_{2}\right)^{01} U_{2}+\left[\left(\log \beta h_{1}\right)^{02}-4 A^{2}\right. \\
& \left.+\left(\log \beta h_{1}\right)^{01}\left(\log \beta^{2} h_{1}\right)^{01}\right] U_{1} \\
& -4 A^{2}(\log A \beta)^{01} U+4 \gamma B^{2} V-\gamma\left(\log \gamma k_{1}\right)^{10} V_{1}-\gamma V_{2}=0
\end{aligned}
$$

and

$$
\begin{aligned}
V_{3} & +\left(\log \gamma{ }^{3} k_{1}^{2} k_{2}\right)^{10} V_{2}+\left[\left(\log \gamma k_{1}\right)^{20}-4 B^{2}\right. \\
& \left.+\left(\log \gamma k_{1}\right)^{10}\left(\log \gamma^{2} k_{1}\right)^{10}\right] V_{1} \\
& -4 B^{2}(\log B \gamma)^{10} V+4 \beta A^{2} U-\beta\left(\log \beta h_{1}\right)^{01} U_{1}-\beta U_{2}=0 .
\end{aligned}
$$

1 See Godeaux, La theorie des surfaces et l'espace régle, 1934, Paris. A direct definition of the sequence of Godeaux quadrics has been given by the present author. See S. C. Chang, Some theorems on ruled surfaces, in the press of Science Records, Academia Sinica.

${ }^{2}$ L. Godeaux, loc. cit. 
If four consecutive asymptotic $u$-tangents $(v=$ const.) belong to a regulus, then there is a plane in $S_{5}$ which intersects the surface $(U)$ at four consecutive points. In other words: $U, d U, d^{2} U, d^{3} U$ should be coplanar.

By means of (3), (4), (5) and (6) it is clear that the three points

$$
\begin{gathered}
\beta V d u+U_{1} d v \\
V\left[\left(\beta^{10}+\beta(\log \gamma)^{10}\right)(d u)^{2}+\beta^{01} d u d v+\beta d^{2} u\right] \\
+\beta V_{1}(d u)^{2}+U_{1} d^{2} v+\left[U_{2}+U_{1}\left(\log \beta h_{1}\right)^{01}\right](d v)^{2}
\end{gathered}
$$

and

$$
\begin{aligned}
& {\left[U_{3}-4 A^{2}(\log A \beta)^{01} U\right](d v)^{3}+M U_{1}+\beta V_{2}(d u)^{3}+N V} \\
& \quad+V_{1}\left[\beta\left(\log \gamma^{2} k_{1}\right)^{10}(d u)^{3}+2 \beta^{10}(d u)^{3}+2 \beta^{01} d v(d u)^{2}+3 \beta d u d^{2} u\right] \\
& \quad+U_{2}\left[\left(\log \beta^{2} h_{1}^{2} h_{2}\right)^{01}(d v)^{3}+3 d v d^{2} v\right]
\end{aligned}
$$

are collinear, where

$$
\begin{aligned}
M= & {\left[\left(\log \beta h_{1}\right)^{01}\right]^{2}(d v)^{3}+d^{3} v+h_{1} d u(d v)^{2}+\left(\log \beta h_{1}\right)^{02}(d v)^{3} } \\
& +3\left(\log \beta h_{1}\right)^{01} d^{2} v d v, \\
N= & 3 \beta^{10} d u d^{2} u+(d u)^{3}\left[\beta^{20}+\beta\left((\log \gamma)^{10}\right)^{2}+\beta(\log \gamma)^{20}+2 \beta^{10}(\log \gamma)^{10}\right] \\
& +(d u)^{2} d v\left[2 \beta^{11}+\beta(\log \gamma)^{11}+2 \beta^{01}(\log \gamma)^{10}+\beta k_{1}\right]+\beta^{02} d u(d v)^{2} \\
& +2 \beta^{01} d^{2} u d v+\beta^{01} d u d^{2} v+\beta d^{3} u+3 \beta(\log \gamma)^{10} d^{2} u d u .
\end{aligned}
$$

From (6), (8), (9) and (10) it follows that the coefficient of $V_{2}$ in (10) must vanish, so that

$$
\beta(d u)^{3}+\gamma(d v)^{3}=0 .
$$

Moreover, we demand that the coefficients of $U_{2}, V_{1}$ in (9) should be proportional to those in (10), and therefore that

(12) $d u d^{2} v-d v d^{2} u-(1 / 3) d u d v\left[\left(\log \beta^{2} \gamma\right)^{10} d u+\left(\log \beta^{3}\right)^{01} d v\right]=0$.

Differentiation of (11) shows that

(13) $3\left(d u d^{2} v-d v d^{2} u\right)+(d u)^{2} d v(\log (\gamma / \beta))^{10}+d u(d v)^{2}(\log (\gamma / \beta))^{01}=0$.

If (12) and (13) coincide with each other for any direction of Darboux, then

$$
\left(\log \beta \gamma^{2}\right)^{10}=0, \quad\left(\log \gamma \beta^{2}\right)^{01}=0 .
$$

Thus we may take

$$
\beta=1, \quad \gamma=1,
$$


and accordingly,

$$
N=d^{3} u+(d u)^{2} d v, \quad M=d^{3} v+d u(d v)^{2} .
$$

Hence the surface in consideration must be a surface of coincidence.

We inquire now whether the asymptotic tangents of some one system along a curve on a surface belong to a regulus. It is seen from (11) that the curve is necessarily a Darboux curve. In virtue of (11) and (15) we obtain a point

$$
\begin{aligned}
V\left[d^{3} u+(d u)^{2} d v+(-\right. & \left.\left.4 B^{2}\right)(d v)^{3}-3 d^{2} v d^{2} u / d v\right] \\
& +U_{1}\left[d^{3} v+d u(d v)^{2}+4 A^{2}(d u)^{3}-3\left(d^{2} v\right)^{2} / d v\right] .
\end{aligned}
$$

This is collinear with the points (9) and (10) and coincides with the point (8) when

$$
\begin{aligned}
& d v\left[d^{3} u+(d u)^{2} d v-4 B^{2}(d v)^{3}-3 d^{2} v d^{2} u / d v\right] \\
& -d u\left[d^{3} v+d u(d v)^{2}+4 A^{2}(d v)^{3}-3\left(d^{2} v\right)^{2} / d v\right]=0
\end{aligned}
$$

From (13), (15) and (16) we have

$$
A^{2}=0, \quad B^{2}=0 .
$$

A reference to (15) and (17) shows that the surface must be $x y z=1$ or one of its projective transforms.

National University of Chekiang 